\title{
Anomalies de la croissance du tissu parathyroïdien dans I'hyperparathyroïdie primaire et secondaire
}

L'hyperplasie du tissu parathyroïdien constitue une anomalie fréquente et parfois grave qui nécessite souvent une cure chirurgicale. Les maladies le plus communément impliquées sont l'hyperparathyroïdie primitive sporadique - soit par adénome d'une seule glande, soit par hyperplasie diffuse des quatre glandes - et l'hyperparathyroïdie secondaire à l'insuffisance rénale chronique.

On sait depuis plusieurs années que le mode de croissance de l'adénome parathyroïdien primaire est celui d'une prolifération monoclonale bénigne [1]. Bien que le (ou les) facteur(s) impliqué(s) ne soient pas encore parfaitement identifiés dans tous les cas, des réarrangements de l'oncogène PRAD1/cycline D1 ont été observés au moins dans un sous-groupe des malades atteints d'adénome $\left(\mathrm{m} / \mathrm{s} n^{\circ} 6\right.$, vol. $7, p$. 635). Une croissance de type monoclonal a également été démontrée dans l'hyperplasie parathyroïdienne liée à la polyendocrinopathie familiale de type I (MEN-1) et la perte allélique d'un gène supposé suppresseur, de localisation 1 lq13, a été rapportée $[1,2]\left(m / s n^{\circ} 6\right.$, vol. 7, p. 635).

En revanche, le mode de croissance des anomalies parathyroïdiennes multiglandulaires non familiales n'avait pas encore fait l'objet d'une analyse systématique jusqu'à une date récente. Une croissance de type polyclonal était généralement tenue pour responsable de ces hyperplasies touchant toutes les parathyroïdes, probablement en réponse à un ou plusieurs stimuli d'origine systémique, mais une étude précise du type de prolifération faisait défaut. Arnold et al. [3] viennent de combler cette lacune. Ils ont examiné le mode de croissance du tissu parathyroïdien dans l'hyperparathyroïdie primitive sporadique (non familiale) par hyperplasie des quatre glandes, d'une part, et dans l'hyperparathyroïdie secondaire à l'insuffisance rénale, d'autre part. Ils ont eu recours à la technique de l'analyse de l'inactivation du chromosome $\mathrm{X}$, en limitant nécessairement leur étude aux sujets de sexe féminin. Cette technique consiste à étudier l'état de méthylation d'un site sensible à l'enzyme Hpall dans la région M27 $\beta$ du chromosome $X$. Ce site critique est méthylé lorsqu'il se trouve sur le chromosome $\mathrm{X}$ activé alors qu'il est non méthylé sur le chromosome $\mathrm{X}$ inactivé. Dans un tissu caractérisé par une croissance monoclonale, c'est-àdire dérivé d'une seule cellule progénitrice, on trouvera un type de clivage très préférentiel par HpaII, puisque le mode d'inactivation de l'X aura été transmis à toutes les cellules analysées.

En utilisant cette technique, les auteurs ont montré que $38 \%$ des cas d'hyperplasie parathyroïdienne primaire de type sporadique présentaient une croissance de type monoclonal, et $64 \%$ des cas d'hyperplasie parathyroïdienne secondaire à l'insuffisance rénale (malades hémodialysés). Il n'a pas été trouvé de correspondance entre l'aspect histologique et la clonalité des tissus examinés. Il faut noter que l'étude d'échantillons tissulaires de quatorze glandes parathyroïdes normales n'a jamais montré d'échantillon monoclonal, éliminant, pour ce petit organe, la possibilité qu'il dérive parfois d'une seule cellule progénitrice. Une mutation d'un ou de plusieurs gènes somatiques est probablement à l'ori- gine de la transformation tumorale bénigne. Il reste à identifier la nature d'une telle mutation. Une perte allélique du locus llq13 était présente chez l'un des quatre malades informatifs atteints d'une hyperplasie parathyroïdienne primaire mais dans aucune des dix-huit glandes informatives provenant des malades urémiques. Un gène suppresseur localisé sur le chromosome $\mathrm{X}$ pourrait être impliqué.

La transformation d'une croissance polyclonale vers une croissance monoclonale d'allure tumorale représente vraisemblablement une étape importante dans l'évolution des hyperplasies parathyroïdiennes, primaires et secondaires.

T.B.D.

1. Arnold A. Genetic basis of endocrine disease 5 Molecular genetics of parathyroid gland neoplasia. J Clin Endocrinol Metab 1993 ; 77: $1108-12$. 2. Calmettes C. Le cancer médullaire de la thyroide: acquis et interrogations. méderine/sciences $1991 ; 7: 22-9$.

3. Arnold A, Brown MF, Urena P, Gaz. RD, Sarfati E, Drüeke TB. Monoclonality of parathyroid tumors in chronic renal failure and in primary parathyroid hyperplasia. J Clin Invest $1995 ; 95$ : 2047-54. 